\section{Jugular valve function and petrosal sinuses pressure: a computational model applied to sudden sensorineural hearing loss}

\author{
Mirko Tessari, ${ }^{1}$ Andrea Ciorba, ${ }^{2}$ \\ Lucas Omar Mueller,, ${ }^{3,4}$ Qinghui Zhang, ${ }^{3}$ \\ Mariapaola Cristini, ${ }^{3}$ Erica Menegatti, ${ }^{1}$ \\ Manuela Mazzoli, ${ }^{2}$ Stefano Pelucchi, ${ }^{2}$ \\ Eleuterio Francisco Toro ${ }^{3}$ \\ 'Vascular Diseases Center, University of \\ Ferrara, Italy; ${ }^{2}$ Department of \\ Biomedical Sciences and Specialty \\ Surgery, Section of Otorinolaringology, \\ University of Ferrara, Italy; \\ ${ }^{3}$ Laboratory of Applied Mathematics, \\ University of Trento, Trento, TN, Italy; \\ ${ }^{4}$ National Laboratory of Computing \\ Sciences, Petrópolis, Rio de Janeiro, \\ Brazil
}

\begin{abstract}
Reports of extra-cranial venous outflow disturbances have recently been linked to sudden sensorineural hearing loss (SSNHL). Aims of the present study are: i) to quantify, with mathematical model, the impact of jugular valve function on the pressure of the superior and inferior petrosal sinuses (SPS, IPS) and the main auricolar veins; ii) to verify the feasibility of the application of mathematical model in the clinical setting in terms of consistency respect to the usual measures of SSNHL outcome. Extra-cranial venous outflow and post analysis were respectively blindly assessed by echo colour-Doppler (ECD) and a validated mathematical model for the human circulation. The pilot study was conducted on 1 healthy control and in a group of 4 patients with different outcome of SSNHL. The main finding was the significant increased pressure calculated in the SPS and IPS of patients with ipsilateral jugular obstruction due to not mobile valve leaflets $(6.55 \mathrm{mmHg})$, respect to the other subjects without extracranial complete obstruction $(6.01 \mathrm{mmHg}), \quad \mathrm{P}=0.0006$. Moreover, we demonstrated an inverted correlation between the extrapolated pressure values in the SPS/IPS and the mean flow measured in the correspondent internal jugular vein $(\mathrm{r}=-0.87773$; r-squared $=$ $0.7697 ; \mathrm{P}=0.0009)$. The proposed mathematical model can be applied to venous extra-cranial ECD investigation in order to derive novel clinical information on the
\end{abstract}

drainage of the inner ear. Such clinical information seems to provide coherent parameters potentially capable to drive the prognosis. This innovative approach was proven to be feasible by the present pilot investigation and warrants further studies with an increased sample of patients.

\section{Introduction}

Chronic cerebrospinal venous insufficiency (CCSVI) is a condition characterized by outflow obstruction in the extracranial venous system mainly caused by intraluminal obstacles, defective valves, hypoplasia, and/or compression of the internal jugular veins (IJV) and/or Azygos vein. ${ }^{1-4}$ This condition was initially brought forth as a possible contributing factor to the pathogenesis and clinical manifestations of multiple sclerosis $^{2-4}$ and, quite recently, of other neurodegenerative diseases, including Alzheimer, ${ }^{5}$ Parkinson, ${ }^{6}$ Ménière disease, ${ }^{7-10}$ and quite anedoctically, to sudden sensorineural hearing loss (SSNHL). ${ }^{11,12}$

SSNHL can be defined as a sensorineural hearing loss of $30 \mathrm{~dB}$ or greater over at least three contiguous audiometric frequencies occurring within a $72-\mathrm{h}$ period. ${ }^{13,14}$ It is an acute inner ear disorder, mostly unilateral. It has been reported that SSNHL has a reported overall incidence of 5-20/100,000 and that is more frequent in western countries. ${ }^{11}$ Despite individuals of all ages can be affected, the peak incidence is reported between the fifth and sixth decade of life and occurs with same incidence in men and women. ${ }^{13,14}$

The pathogenesis of SSNHL is still unknown; viral infections and vascular occlusions are the more common mechanisms advocated. Other reported causes of SSNHL include infectious, autoimmune, traumatic, vascular, neoplastic, metabolic and neurologic diseases. ${ }^{13-16}$ The vascular hypothesis of SSNHL pathogenesis seems the more promising, albeit clinical information about consequences of extracranial venous outflow disturbances in the main intracranial route of drainage of the inner ear is currently unavailable. ${ }^{17,18}$ Alternatively, computation offers nowadays the possibility of quantifying brain haemodynamics. ${ }^{19-22}$

Aims of the present study are: i) to quantify, by means of an existing mathematical model, ${ }^{19}$ the impact of extracranial cerebral venous outflow on bilateral pressure of the superior and inferior petrosal sinuses (SPS, IPS), posterior auricular, deep facial, mastoid emissary, IJV; ii) to verify the feasibility of the application of the
Correspondence: Mirko Tessari, Vascular Diseases Center, University of Ferrara, via Aldo Moro 8, 44124 Cona (FE), Italy. Tel.: +39.0532.239498 - Fax +39.0532.239788 E-mail: mirko.tessari@unife.it

Key words: Sudden sensorineural hearing loss; cerebral venous return; duplex; internal jugular vein; vascular ultrasound; computational model; chronic cerebrospinal venous insufficiency.

Conflict of interest: the authors declare that they have no conflict of interest.

Received for publication: 15 March 2017

Revision received: 5 April 2017.

Accepted for publication: 8 April 2017.

This work is licensed under a Creative Commons Attribution 4.0 License (by-nc 4.0).

(C) Copyright M. Tessari et al., 2017

Licensee PAGEPress, Italy

Veins and Lymphatics 2017; 6:6707

doi:10.4081/vl.2017.6707

mathematical model in the clinical setting in terms of consistency respect to the usual measures of SSNHL outcome.

\section{Materials and Methods}

The pilot study was blindly conducted on 1 healthy control (HC) (male 60 y.o.) and in a group of 4 patients with different outcome of SSNHL (mean age 69 y.o. and ratio male:female 1:1).

The study was approved by the Ethical Committee of the Azienda OspedalieroUniversitaria of Ferrara (Italy), with the registry number \#101298 All patients and $\mathrm{HC}$ signed an informed consent.

\section{Ultrasonographic study of cerebral venous return}

The five subjects were blindly investigated in the vertebral veins (VVs) and in the IJVs, through an echo color Doppler (ECD) protocol recommended by an international consensus for assessing flow disturbances of cerebral venous return (Esaote MyLab 70, Probe $7.510 \mathrm{mHz}$, Genoa, Italy)..$^{20,21}$ This protocol includes also a M-mode study of the jugular valve motility. ${ }^{23}$

In this study we focused the attention on the junctional area where a valve is present in about $60 \%$ normal subjects. ${ }^{23}$ If an abnormal valve was detected according to the criteria of adopted protocol the flow characteristic were deeply investigated measuring not only flow direction but also peak veloc- 
Table 1. Clinical and demographic characteristic of the patients affected by SSNHL.

\begin{tabular}{llccccc} 
Subject & Age & Gender & SSNHL side & $\begin{array}{c}\text { Responder } \\
\text { to treatment }\end{array}$ & $\begin{array}{c}\text { Clinical } \\
\text { consistency } \\
\text { consistency } \\
\text { (recovery and SPS/ } \\
\text { IPS pressure) }\end{array}$ \\
SSNHL \#1 & 73 y.0. & F & Right & No recovery & Negative & Negative \\
SSNHL \#2 & 54 y.0. & F & Right & No recovery & Positive & Positive \\
\hline SSNHL \#3 & 71 y.0. & M & Right & No recovery & Positive & Positive \\
SSNHL \#4 & 79 y.0. & M & Left & Recovery & Negative & Positive \\
\hline
\end{tabular}

ity, time average velocity, flow and cross sectional area. ${ }^{24-26}$

Subsequently, we assigned, on the bases of the ECD investigation, a non-dimensional score of the valve status. This function varies between 0 and 1 , and represents the rate of opening and closing of the valve; the 0 score represents a normal valve function and whose assigned when the flow was monodirectional with the values of the above haemodynamic parameters within range of normality. ${ }^{26-29}$ We assigned a score of 0.25 when the flow was bi-directional, although prevalently directed outward the chest, in consequence of a not mobile single leaflet. Moreover, we assigned a score of 0.50 when the flow was completely bidirectional and/or when the defective valve increases the flow velocity $>120 \mathrm{~cm} / \mathrm{sec}$; the score was 0.75 if the flow was bi-directional but prevalently directed inward the brain and/or if the flow velocity was greater than $150 \mathrm{~cm} / \mathrm{sec}$. Finally, in case of complete collapse of the lumen and/or absence of detectable flow, and/or not mobile valve with the flow unable to cross the intraluminal obstacle, we assigned a score of 1 .

\section{Application of the computational model}

The non-dimensional number expressing the valve state, above reported, depends on a time-dependent function $\xi(\mathrm{t})$ which is given by the solution of variable-coefficient ordinary differential equations:

$\frac{d}{d t} \xi(t)=\left\{\begin{array}{cl}K_{\odot}\left[\Delta p(t)-\Delta p_{e}\right](1-\xi(t)) & \text { if } \Delta p(t)>\Delta p_{\bullet} \text { (opening), } \\ K_{e}\left[\Delta p(t)-\Delta p_{e}\right] \xi(t) & \text { if } \Delta p(t)<\Delta p_{e} \text { (closing). }\end{array}\right.$

The non-dimensional valve state, with $0 \leq \xi(t) \leq 1$, represents the rate respectively of opening and closing of the IJV valve. $\mathrm{K}_{\mathrm{o}}$ is the coefficient for opening the valce with $\Delta \mathrm{p}_{\mathrm{o}}$ who representing the threshold pressure to open the leaflets of the valve. $\mathrm{K}_{\mathrm{c}}$ is a coefficient to close the valve with $\mathrm{Dp}_{\mathrm{c}}$ who acting for the threshold pressure for closing the leaflets. Dp(t) is the pres-

Table 2. Score assigned for intracranial computational analysis after high resolution Doppler sonography study of the valvular region of the IJVs.

\begin{tabular}{lcccc} 
Subjects & RIJ & LJV & RWV & LV \\
HC & 0 & 0 & 0 & 0 \\
SSNHL \#1 & 0.25 & 0.75 & 1.0 & 1.0 \\
\hline SSNHL \#2 & 1.0 & 0 & 0 & 0 \\
SSNHL \#3 & 1.0 & 0 & 0 & 1.0 \\
\hline SSNHL \#4 & 0.25 & 0.25 & 0 & 0 \\
\hline
\end{tabular}

sure variation of time-dependent function.

Computational analysis was blindly performed respect to the clinical outcome on the basis of the ECD assessment and score of the IJV valve function, as described above. Extrapolation of the intracranial haemodynamics was achieved by applying a global mathematical model for the human circulation, previously validated against in vivo MRI data. ${ }^{19}$ We performed five sets of simulations for each subject in supine position.

\section{Clinical outcome evaluation}

The clinical side of SSNHL at onset, as well as the prognosis and response to treatment (oral steroid therapy accordingly to the AAO-HNS indications), ${ }^{29}$ were blindly assessed by the treating physician. The consistency of the computational parameters extrapolated at the level of the draining veins of the hearing apparatus with side and clinical outcome were subsequently analyzed.

\section{Statistical analysis}

The extrapolated pressure values in the SPS/IPS of subjects with complete obstruction of an extracranial venous segment were tested for significance vs the pressure values of people with score flow between 0 and 0.75 by the means of two-tailed MannWhitney test.

The value of flow in the IJVs were correlated with SPS/IPS pressure by the means of linear regression analysis. Value $<0.05$ were considered significant.

\section{Results}

In Table 1 patients' demographic and clinical characteristics are given, including the side and the clinical outcome of SSNHL. Our cases reflect the male:female ratio of the disease (1:1). Moreover patients $\# 1, \# 2, \# 3$ were affected by SSNHL on the right side, and just patient $\# 4$ on the left one.

It is worth of note that in patients \#2, \#3, the abnormal right valve of the IJV determined absence of flow through the junction with consequent assignment of non-dimensional score 1 . The worse score was also given to patient \#1 for absence of Doppler detectable flow in both vertebral veins. Finally, in patient \#4 few and not significant abnormalities were detected along the major brain outflow routes. The nondimensional score assigned in accordance with ECD findings are summarized for all subjects in Table 2 .

In Table 3 we give the complete detail of flow, velocity, pressure and cross sectional area extrapolated by computational analysis in the major anatomic draining pathways of the inner ear region. Particularly, in the SPS and IPS, which both represent the major intracranial draining route, the calculated value of pressure were significantly more elevated on the right side, in patients $\# 2$, \#3, as compared to both the rest of the patients and the control. In Figure 1 the calculated pressure is very well apparent because the curves of patients \#2, \#3 are overlapped, showing a higher course respect the curves of the other subjects. For 
patient \#1 the computational model, despite the score 1 assigned to both $\mathrm{VVs}$, did not calculate any significant pressure increment in SPS/IPS. This because the global model utilized takes into account the anatomical and physiological connections among the different vessels.

Despite the small sample, the pressure in the SPS/IPS of subjects with score 1 in one or more of the investigated outflow route, is significantly higher $(6.55 \mathrm{mmHg})$ respect to the other subjects without extracranial complete obstruction (6.01 $\mathrm{mmHg}$ ), $\mathrm{P}=0.0006$ (Table 3). Moreover, in Figure 2 we show the inverted correlation between the extrapolated pressure values in the SPS/IPS of the 5 subjects respect to the mean flow measured in the correspondent IJVs $(r=-0.87773$; $r$ squared $=0.7697$; $\mathrm{P}=0.0009$ ). The strong correlation found clearly indicates how the pressure increase in the SPS/IPS is dependent from the hampered IJV flow at the extracranial level.

\section{Discussion and Conclusions}

The pathogenesis of SSNHL is still unknown; viral infections and vascular disease are the more common mechanisms advocated, even if evidence in favour of a specific mechanism is still insufficient. ${ }^{30}$ Of the possible causes of SSNHL, vascular involvement has captured much attention, and some studies in the literature have investigated the association between
SSNHL and vascular disease to date. A better understanding of the relationship between cochlear blood flow and hearing function is fundamental for improving the treatment and diagnosis of deafness that potentially arises from circulatory abnormalities. Achieving such understanding has been challenging because of the experimental difficulties involved in monitoring cochlear blood flow. ${ }^{30}$ However, computa-
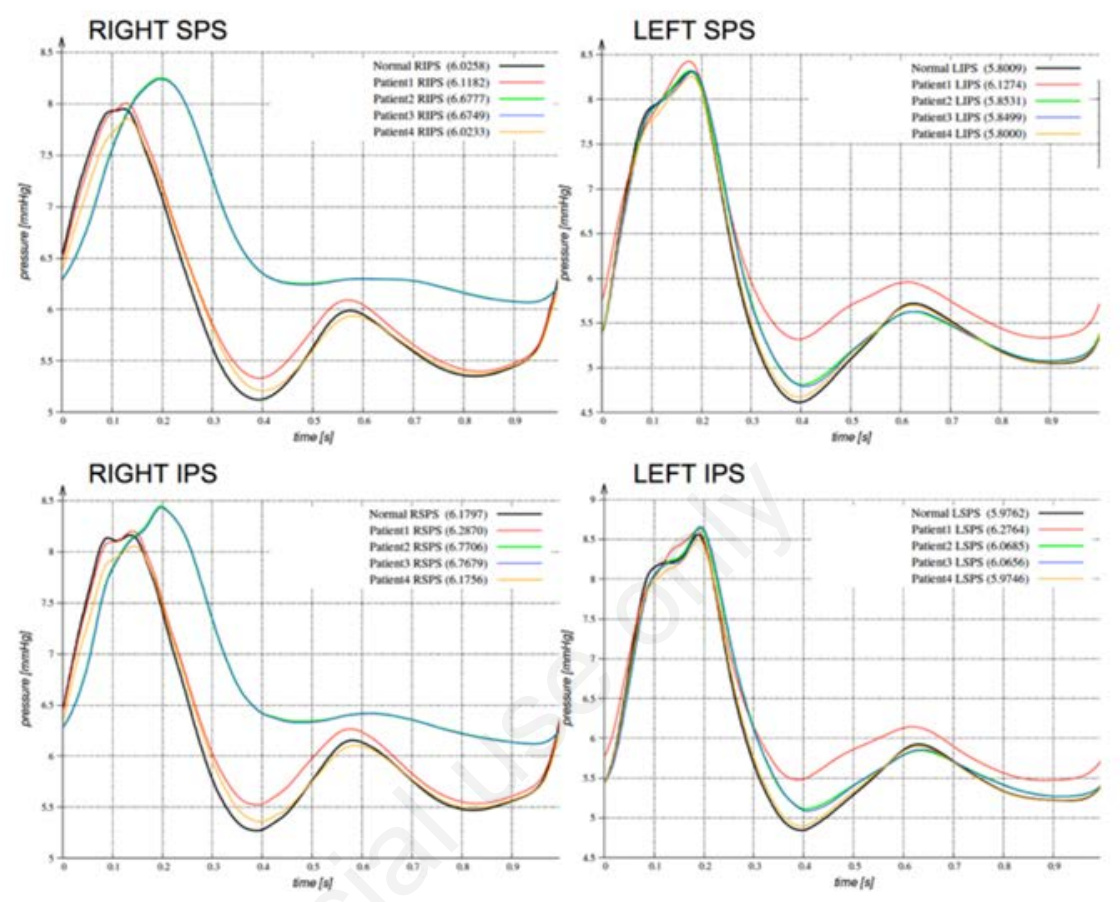

Figure 1. Right graphs: The increased pressure calculated on the right superior and inferior Petrosus sinus in patients with blocked flow at the level of the right IJV valve (\#2, \#3) is well apparent as compared the rest of the cohort. Left graphs: substantial overlapping of the pressure curves calculated on the left SPS/IPS in all subjects.

Table 3. Computational extra-intracranial venous haemodynamics calculated in patients and control.

\begin{tabular}{|c|c|c|c|c|c|c|c|c|c|c|c|c|c|}
\hline Subject & & $\begin{array}{c}\text { R posterior } \\
\text { auricular } \\
\text { V }\end{array}$ & $\begin{array}{c}\text { L posterior } \\
\text { auricular } \\
\text { V }\end{array}$ & $\begin{array}{l}\text { R deep } \\
\text { facial } \\
\mathrm{V}\end{array}$ & $\begin{array}{l}\text { L deep } \\
\text { facial } \\
\mathrm{V}\end{array}$ & $\begin{array}{c}\text { R mastoid } \\
\text { emissary } \\
V\end{array}$ & $\begin{array}{l}\text { L mastoid } \\
\text { emissary } \\
\text { V }\end{array}$ & $\begin{array}{l}\text { R superior } \\
\text { petrosal } \\
\text { sinus }\end{array}$ & $\begin{array}{l}\text { L superior } \\
\text { petrosal } \\
\text { sinus }\end{array}$ & $\begin{array}{l}\text { R inferior } \\
\text { petrosal } \\
\text { sinus }\end{array}$ & $\begin{array}{l}\text { Linferior } \\
\text { petrosal } \\
\text { sinus }\end{array}$ & $\begin{array}{l}\text { R internal } \\
\text { jugular } \\
\mathrm{V}\end{array}$ & $\begin{array}{c}\text { L internal } \\
\text { jugular } \\
\mathrm{V}\end{array}$ \\
\hline $\mathrm{HC}$ & $\begin{array}{l}\text { Flow } \\
\text { Pressure } \\
\text { Velocity } \\
\text { CSA }\end{array}$ & $\begin{array}{l}0.2315 \\
6.8514 \\
0.1126 \\
2.0575\end{array}$ & $\begin{array}{l}0.2315 \\
6.7820 \\
0.1128 \\
2.0557\end{array}$ & $\begin{array}{c}-0.1118 \\
5.9788 \\
-0.0056 \\
20.1318\end{array}$ & $\begin{array}{c}-0.1336 \\
5.9210 \\
-0.0067 \\
20.0886\end{array}$ & $\begin{array}{l}0.3160 \\
6.1262 \\
0.0321 \\
9.8500\end{array}$ & $\begin{array}{l}0.2669 \\
5.8838 \\
0.0274 \\
9.7938\end{array}$ & $\begin{array}{c}-0.1259 \\
6.1797 \\
-0.0192 \\
6.5736\end{array}$ & $\begin{array}{c}-0.0258 \\
5.9762 \\
-0.0039 \\
6.5667\end{array}$ & $\begin{array}{c}-0.0089 \\
6.0258 \\
-0.0015 \\
5.7988\end{array}$ & $\begin{array}{l}0.0894 \\
5.8009 \\
0.0155 \\
5.7921\end{array}$ & $\begin{array}{c}3.6780 \\
5.5954 \\
0.0735 \\
51.0108\end{array}$ & $\begin{array}{c}9.9242 \\
5.6758 \\
0.0817 \\
124.1327\end{array}$ \\
\hline SSNHL \#1 & $\begin{array}{l}\text { Flow } \\
\text { Pressure } \\
\text { Velocity } \\
\text { CSA }\end{array}$ & $\begin{array}{l}0.2306 \\
6.8690 \\
0.1122 \\
2.0579\end{array}$ & $\begin{array}{l}0.2304 \\
6.9898 \\
0.1120 \\
2.0606\end{array}$ & $\begin{array}{c}-0.1114 \\
6.0118 \\
-0.0056 \\
20.1484\end{array}$ & $\begin{array}{c}-0.1713 \\
6.1847 \\
-0.0086 \\
20.2253\end{array}$ & $\begin{array}{l}0.3351 \\
6.2204 \\
0.0340 \\
9.8693\end{array}$ & $\begin{array}{l}0.2630 \\
6.2099 \\
0.0267 \\
9.8640\end{array}$ & $\begin{array}{c}-0.1167 \\
6.2870 \\
-0.0178 \\
6.5687\end{array}$ & $\begin{array}{c}-0.0644 \\
6.2764 \\
-0.0098 \\
6.5684\end{array}$ & $\begin{array}{l}0.0373 \\
6.1182 \\
0.0065 \\
5.7941\end{array}$ & $\begin{array}{l}0.0350 \\
6.1274 \\
0.0060 \\
5.7944\end{array}$ & $\begin{array}{c}4.2219 \\
5.5636 \\
0.0845 \\
50.9385\end{array}$ & $\begin{array}{c}8.3219 \\
5.6530 \\
0.0687 \\
123.9612 \\
\end{array}$ \\
\hline SSNHL \#2 & $\begin{array}{l}\text { Flow } \\
\text { Pressure } \\
\text { Velocity } \\
\text { CSA }\end{array}$ & $\begin{array}{l}0.2302 \\
7.4177 \\
0.1112 \\
2.0708\end{array}$ & $\begin{array}{l}0.2311 \\
6.8106 \\
0.1125 \\
2.0564\end{array}$ & $\begin{array}{c}-0.2066 \\
6.6517 \\
-0.0101 \\
20.4590\end{array}$ & $\begin{array}{c}-0.1274 \\
5.9589 \\
-0.0064 \\
20.1092\end{array}$ & $\begin{array}{l}0.2684 \\
6.7689 \\
0.0269 \\
9.9735\end{array}$ & $\begin{array}{l}0.2874 \\
5.9331 \\
0.0295 \\
9.8051\end{array}$ & $\begin{array}{c}-0.1768 \\
6.7706 \\
-0.0269 \\
6.5919\end{array}$ & $\begin{array}{l}0.0376 \\
6.0685 \\
0.0058 \\
6.5680\end{array}$ & $\begin{array}{c}-0.1404 \\
6.6777 \\
-0.0242 \\
5.8168\end{array}$ & $\begin{array}{l}0.2141 \\
5.8531 \\
0.0371 \\
5.7920\end{array}$ & $\begin{array}{c}0.1946 \\
5.5792 \\
0.0039 \\
50.9774\end{array}$ & $\begin{array}{c}11.5439 \\
5.6440 \\
0.0951 \\
123.9877\end{array}$ \\
\hline SSNHL \#3 & $\begin{array}{l}\text { Flow } \\
\text { Pressure } \\
\text { Velocity } \\
\text { CSA }\end{array}$ & $\begin{array}{l}0.2301 \\
7.4146 \\
0.1112 \\
2.0708\end{array}$ & $\begin{array}{l}0.2310 \\
6.8062 \\
0.1125 \\
2.0563\end{array}$ & $\begin{array}{c}-0.2063 \\
6.6491 \\
-0.0101 \\
20.4579\end{array}$ & $\begin{array}{c}-0.1272 \\
5.9548 \\
-0.0064 \\
20.1076\end{array}$ & $\begin{array}{l}0.2684 \\
6.7661 \\
0.0269 \\
9.9730\end{array}$ & $\begin{array}{l}0.2866 \\
5.9303 \\
0.0294 \\
9.8043\end{array}$ & $\begin{array}{c}-0.1768 \\
6.7679 \\
-0.0269 \\
6.5914\end{array}$ & $\begin{array}{l}0.0373 \\
6.0656 \\
0.0057 \\
6.5675\end{array}$ & $\begin{array}{c}-0.1404 \\
6.6749 \\
-0.0242 \\
5.8163\end{array}$ & $\begin{array}{l}0.2142 \\
5.8499 \\
0.0371 \\
5.7916\end{array}$ & $\begin{array}{c}0.1953 \\
5.5719 \\
0.0040 \\
50.9660\end{array}$ & $\begin{array}{c}11.5997 \\
5.6394 \\
0.0956 \\
123.9529 \\
\end{array}$ \\
\hline SSNHL \#4 & $\begin{array}{l}\text { Flow } \\
\text { Pressure } \\
\text { Velocity } \\
\text { CSA }\end{array}$ & $\begin{array}{l}0.2309 \\
6.8294 \\
0.1123 \\
2.0571 \\
\end{array}$ & $\begin{array}{l}0.2308 \\
6.7710 \\
0.1124 \\
2.0555 \\
\end{array}$ & $\begin{array}{c}-0.1123 \\
5.9624 \\
-0.0056 \\
20.1278 \\
\end{array}$ & $\begin{array}{c}-0.1352 \\
5.9157 \\
-0.0068 \\
20.0884 \\
\end{array}$ & $\begin{array}{l}0.3144 \\
6.1242 \\
0.0319 \\
9.8510\end{array}$ & $\begin{array}{l}0.2665 \\
5.8828 \\
0.0273 \\
9.7948 \\
\end{array}$ & $\begin{array}{c}-0.1286 \\
6.1756 \\
-0.0196 \\
6.5743\end{array}$ & $\begin{array}{c}-0.0269 \\
5.9746 \\
-0.0041 \\
6.5675 \\
\end{array}$ & $\begin{array}{c}-0.0092 \\
6.0233 \\
-0.0016 \\
5.7995\end{array}$ & $\begin{array}{l}0.0883 \\
5.8000 \\
0.0153 \\
5.7928 \\
\end{array}$ & $\begin{array}{l}3.7311 \\
5.5671 \\
0.0746 \\
50.9560\end{array}$ & $\begin{array}{c}9.8364 \\
5.6510 \\
0.0811 \\
123.9738 \\
\end{array}$ \\
\hline
\end{tabular}


tion analisys could offer nowadays the possibility of quantifying brain haemodynamics. In particular, we have applied a validated protocol of ECD evaluation of the extracranial veins to a confirmed computational model in order to assess haemodynamic parameters in the vein of the inner ear, and particularly in the major dural sinuses draining it. The main finding of our study is the increased pressure calculated by the clinical application of the model at the level of the right petrosus sinuses in patients presenting ECD with significantly compromised valve function at the level of the IJV junction. The curves comparing SPS/IPS pressure in patients and control (Figure 1) clearly show the raise in venous pressure, at the intracranial level, of patients exhibiting absence of Doppler detectable flow through the defective valve. . $3,23,24,31,32^{2}$

The four patients presented some abnormalities in cerebral venous return, mainly focused on IJV valve function. The valve leaflets motility were M-mode investigated together with spectral-flow characteristics, ${ }^{23}$ demonstrating how the presence of a defective valve opening determines also abnormal haemodynamics. This finding is particularly relevant as it is consistent with both the side of the SSNHL and the clinical outcome. When we calculated a significant increase of the SPS/IPS pressure this corresponded to the side of hearing loss; even more intriguing is the observation that the increased pressure in the dural sinuses is also corresponding to absence of recovery with the current treatment in the same cases (Table 1). Conversely, when we compared the clinical outcome of the patient with no increased pressure in the petrosus sinuses (patient \#4) we found him responder to the treatment. This seems to indicate the potential use of this approach as a clinical prognostic factor. Also we analyzed the consistency of our ECD/computational model results with both the side of SSNHL and clinical outcome (Table 1). As far as the side of onset of the disease is concerned the increased SPS/IPS pressure side corresponds to the side of SSNHL in patients \#2 and \#3. Regarding the clinical outcome, again hyper-pressure in the SPS/IPS seems an important prognostic factor because recovery was achieved in patient \#4 with level of pressure comparable to control, but not in patients \#2 and \#3.

The application of the computational model to the study of the drainage of the inner ear apparently may add a novel potential prognostic factor, particularly interesting in clinical practice. If little is known on the pathogenetic mechanisms that cause SSNHL, even less information is available on the biological mechanism of threshold recovery. It has been reported that the SSNHL threshold-recovery can be in relation to many factors, such as: i) the hearing loss length prior to therapy; ii) the associated hearing loss symptoms; and iii) the audiogram features. It has also been reported that patients with higher hearing loss, at the SSNHL onset, show a lower recovery rate in comparison to patients with initial mild losses..$^{33,34}$ Among the audiogram shape, data from the literature show that patients with low-frequency or mid-frequency hearing losses present higher rates of recovery in comparison to patients with flat or sloping-down audiometric profiles. ${ }^{33-}$ ${ }_{35}$ Computational increased SPS/IPS pressure might be an additional factor because in this pilot study individuated patients with worse prognosis. The raising pressure of the dural sinuses seems to be a pivotal parameter with changes in case of abnormal jugular valve function. The latter can be non-invasively derived by means of ECD.

Quite recently another model of the intra and extracranial circulation applied to clinical cases demonstrated the correlation between increased dural sinuses pressure and clinical outcome in multiple sclerosis patients presenting also extracranial venous obstacles. ${ }^{20}$

Possible shortcomings of our study are linked with the variability of ECD applied to the study cerebral venous return. Several meta-analysis show big variations depending from training and operator dependency of ultrasound. ${ }^{1}$

Surely there is an incidence of CCSVI in patients with SSNHL, but some authors show only $21.6 \%$ of the evaluated cases. ${ }^{12}$ This difference compared to our study, where we have 4 SSNHL patients positive to the diagnoses of CCSVI, it is explicable on the grounds that our population has been identified for the application of the mathematical model with different areas of stenosis and valvular abnormalities. The small sample size does not give us the opportunity to give an incidence of CCSVI.

An impaired cochlear perfusion is widely reported to be a possible cause of SSNHL. Several data support this hypothesis. Firstly, the cochlea has a terminal capillary bed and is more susceptible to impaired drainage and increased pressure respect to regions with available collateral vessels. Secondly, since cochlear hair cells have a

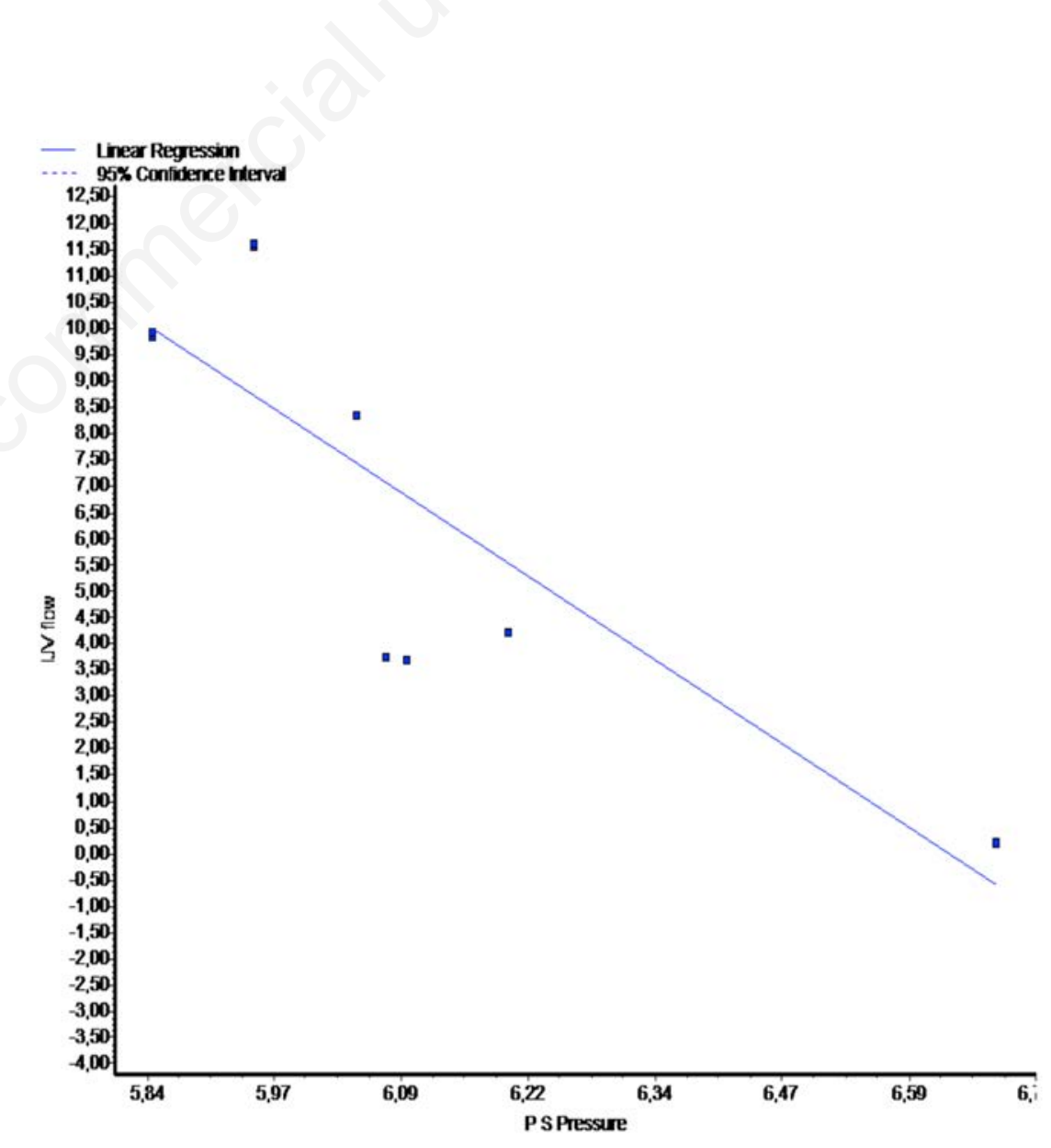

Figure 2. Inverted relationship between IJV flow rate and pressure at the level of the SPS/IPS Petrosus sinuses ( $r=-0.87773 ; \mathrm{r}$ squared $=0.7697 ; \mathrm{p}=0.0009)$. 
high metabolic activity, they are particularly vulnerable to reduced perfusion. ${ }^{33,36}$ Thirdly, several studies have found that risk factors for the endothelial cells, such as cigarette smoking, hypertension, and hyperlipidemia, are also risk factors for the development of SSNHL. ${ }^{35-37}$ Defective IJV valves, similar to those detected by means ECD in this study, have significantly been reported to concur to the injury of the endothelial cells, suggesting a possible correlation with the above environmental risk factors. $^{38}$

Since the present results are encouraging, the next step will consist in applying the combination of ECD extracranial venous outflow assessment coupled with computational analysis in a large sample of patients affected by SSNHL in order to verify the prognostic value of our innovative approach on a large case series.

\section{References}

1. Zivadinov R, Bastianello S, Dake $\mathrm{MD}$, et al. Recommendations for multimodal noninvasive and invasive screening for detection of extracranial venous abnormalities indicative of chronic cerebrospinal venous insufficiency: a position statement of the international society of neurovascular disease. J Vasc Interv Radiol 2014;25:1785-94.

2. Zamboni P, Galeotti P, Menegatti E, et al. Chronic cerebrospinal venous insufficiency in patients with multiple sclerosis. J Neurol Neurosurg Psychiatry 2009;80:392-99.

3. Zivadinov R, Marr K, Cutter G, et al. Prevalence, sensitivity, and specificity of chronic cerebrospinal venous insufficiency in MS. Neurology 2011;77:13844.

4. Traboulsee AL, Knox KB, Machan L, et al. Prevalence of extracranial venous narrowing on catheter venography in people with multiple sclerosis, their siblings, and unrelated healthy controls: a blinded, case-control study. Lancet 2014;383:138-45.

5. Chung CP, Beggs C, Wang PN, et al. Jugular venous reflux and white matter abnormalities in Alzheimer's disease: a pilot study. J Alzheimers Dis 2014;39:601-9.

6. Liu M, Xu H, Wang Y, et al. Patterns of chronic venous insufficiency in the dural sinuses and extracranial draining veins and their relationship with white matter hyperintensities for patients with Parkinson's disease. J Vasc Surg 2015;61:1511-20.
7. Bruno A, Califano L, Mastrangelo D, et al. Chronic cerebrospinal venous insufficiency in Ménière's disease: diagnosis and treatment. Veins and Lymphatics 2014;3:3854

8. Bruno A, Napolitano MS, Califano G, et al. The Prevalence of chronic cerebro-spinal venous insufficiency in Meniere disease: 24 months follow-up after angioplasty. JVIR 2017;28:38891.

9. Filipo R, Ciciarello F, Attanasio G, et al. Chronic cerebrospinal venous insufficiency in patients with Meniere's disease. Eur Arch Otorhinolaryngol 2015;272:77-82.

10. Lopez-Escamez JA, Carey J, Chung W$\mathrm{H}$, et al. Diagnostic criteria for Meniere's disease. J Vestib Res 2015;25:1-7.

11. Alpini D, Bavera PM, Di Berardino F, et al. Bilateral sudden sensorineural hearing loss and chronic venous cerebrospinal insufficiency: a case report. Phlebology 2013;28:231-3.

12. Attanasio G, Cagnoni L, Masci E, et al. Chronic cerebrospinal venous insufficiency as a cause of inner ear diseases. Acta Oto-Laryngologica 2016;16:1-4.

13. Kuhn M, Heman-Ackah SE, Shaikh JA, Roehm PC. Sudden sensorineural hearing loss: a review of diagnosis, treatment, and prognosis. Trends Amplif 2011;5:91-105.

14. Chau JK, Lin JR, Atashband S, et al. Systematic review of the evidence for the etiology of adult sudden sensorineural hearing loss. Laryngoscope 2010;120:1011-21.

15. Wilson WR, Veltri RW, Laird N, Sprinkl PM. Viral and epidemiologic studies of idiopathic sudden hearing loss. Otolaryngol Head Neck Surg 1983;91:653-8

16. Wilson WR, Byl FM, Laird N. The efficacy of ste- roids in the treatment of idiopathic sudden hearing loss. A double- blind clinical study. Acta OtoLaryngol 1980;106:772-6.

17. Zadeh MH, Storper IS, Spitzer JB. Diagnosis and treatment of suddenonset sensorineural hearing loss: a study of 51 patients. Otolaryngol Head Neck Surg 2003;128:92-8.

18. Byl FM. Sudden hearing loss: eight years' experience and suggested prognostic table. Laryngoscope 1984;94:647-61.

19. Caiazzo A, Montecinos G, Muller LO, et al. Computational haemodynamics in stenotic internal jugular veins. J Math Biol 2015;70:745-72.

20. Gadda G, Taibi A, Sisini F, et al. A new hemodynamic model for the study of cerebral venous outflow. Am J Physiol Heart Circ Physiol 2015;308:217-31.

21. Müller LO, Toro EF. Enhanced global mathematical model for studying cerebral venous blood flow. J Biomech 2014;47:3361-72.

22. Müller LO, Toro EF. A global multiscale mathematical model for the humancirculation with emphasis on the venous system. Int $\mathrm{J}$ Numer Method Biomed Eng 2014;30:681-725.

23. Menegatti E, Tessari M, Gianesini S, et al. Human internal jugular valve Mmode ultrasound characterization. Curr Neurovasc Res 2014;11:149-55.

24. Zamboni P, Morovic S, Menegatti E, et al. Screening for chronic cerebrospinal venous insufficiency (CCSVI) using ultrasound--recommendations for a protocol. Int Angiol 2011;30:571-97.

25. Tromba L, Blasi S, Vestri A, et al. Prevalence of chronic cerebrospinal venous insufficiency in multiple sclerosis: a blinded sonographic evaluation. Phlebology 2015;30:52-60.

26. Zamboni P, Menegatti E, Pomidori L, et al. Does thoracic pump influence the cerebral venous return? J Appl Physiol 2012;112:904-10.

27. Zamboni P, Sisini F, Menegatti E, et al. An ultrasound model to calculate the brain blood outflow through collateral vessels: a pilot study. BMC Neurol 2013;13:81.

28. Thibault P, Lewis W, Niblett $\mathrm{S}$. Objective duplex ultrasound evaluation of the extracranial circulation in multiple sclerosis patients undergoing venoplasty of internal jugular vein stenoses: A pilot study. Phlebology 2015;30:98104.

29. Stachler RJ, Chandrasekhar SS, Archer $\mathrm{SM}$, et al. Clinical practice guideline: sudden hearing loss. Otolaryngol Head Neck Surg 2012;146:S1-35.

30. A. Ciorba A, Faita A, Bianchini C, et al. Arteriopathy and microvascular impairment in Sudden Sensorineural Hearing Loss: Clues from two clinical cases. Hearing Balance Commun 2013;11:8790.

31. Mandolesi S, D'Alessandro A, Ciccone $\mathrm{MM}$, et al. Italian Chronic Cerebrospinal Venous Insufficiency National Epidemiological Observatory methodology and preliminary data. Veins and Lymphatics 2014;3:4707.

32. Zamboni P, Consorti G, Galeotti R, et al. Venous collateral circulation of the extracranial cerebrospinal outflow routes. Curr Neurovasc Res 2009;6:204-12.

33. Kuhn M, Heman-Ackah SE, Shaikh JA, Roehm PC. Sudden sensorineural hear- 
ing loss: a review of diagnosis, treatment, and prognosis. Trends Amplif 2011;15:91-105.

34. Zadeh MH, Storper IS, Spitzer JB. Diagnosis and treatment of suddenonset sensorineural hearing loss: A study of 51 patients. Otolaryngol Head Neck Surg 2003;128:92-8.

35. Aimoni C, Bianchini C, Borin M, et al.
Diabetes, cardiovascular risk factors and idiopathic sudden sensorineural hearing loss: a case-control study. Audiol Neurootol 2010;15:111-5.

36. Capaccio P, Ottaviani F, Cuccarini V, et al. Genetic and acquired prothrombotic risk factors and sudden hearing loss. Laryngoscope 2007;117:547-51.

37. Marcucci R, Alessandrello Liotta A,
Cellai AP, et al. Cardiovascular and thrombophilic risk factors for idiopathic sudden sensorineural hearing loss. J Thromb Haemost 2005;3:929-34.

38. Zamboni P, Tisato V, Menegatti E, et al. Ultrastructure of internal jugular vein defective valves. Phlebology 2015;30: 644-7. 